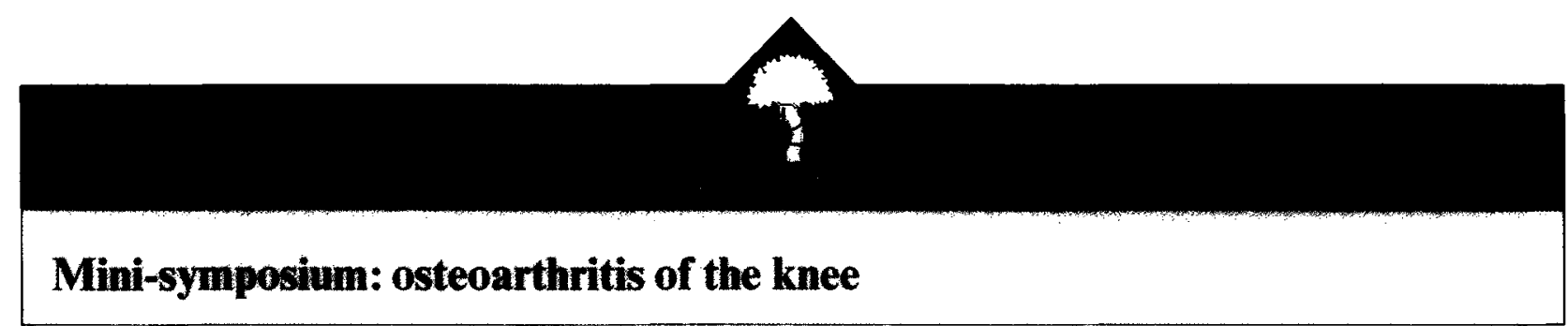

\title{
(v) Surgery for Failed Total Knee Arthroplasty
}

\section{Introduction}

Considerations for surgery for failed total knee arthroplasty are dependent on many factors, but the most important is the presencc or absencc of infection. Many very useful and beneficial options for salvage of a failed knee arthroplasty become irrelevant with a diagnosis of sepsis.

\section{Infection}

Sepsis after total bone replacement may be a fulminant and life-threatening circumstance with systemic manifestations of bacterial proliferation, tissue necrosis, and the effects of related toxins. Frequently infection may be diagnosed during the early postoperative period. Systemic signs in association with wound inflammation and drainage lead to increasing suspicion validated by positive culture results.

While this same fulminant course may occur long after a previously successful replacement arthroplasty, this is not the usual case. More frequently the late infection is seen as a knee that is not doing well. It is painful. swollen and hot. The radiographs show a progressive radiolucent line and there are reactive calcifications or ossifications at the joint capsule.

Since the implications of infection are so important regarding the options for joint salvage, such a knee must be repeatedly aspirated and cultured for all reasonable pathogens before proceeding. Often more than one aspiration will be necessary to obtain true positive results particularly with relatively indolent organisms. Similarly, the prior use of antibiotics may

I.arry S. Matthews MD, University of Michigan, Department of Surgery, Section of Orthopaedic Surgery, 1500 E. Medical Center Drive, Ann Arbor, MI 48109. USA mask or suppress the infection and confound efforts to obtain valid microbiologic results.

While suppressive therapy may decrease the urgency of the situation and allow for protracted compromised function, component loosening usually progresses and it is rare that removal of the prosthesis components and all foreign material can be avoided.

Delay even with antibiotic suppression results in greater soft tissue damage as well as bone loss. Success with repeat prosthetic arthroplasty, resection nonprosthetic arthroplasty, and fusion is partially dependent on sufficient residual metaphyseal bone. With suspicion of infection, sterile cultures should be obtained and repeated until valid results are certain. If the knee is infected prosthetic removal is inevitable and should be done before too much bone loss has occurred.

The optimal salvage for an infected total knee arthroplasty is repeat arthroplasty after infection has been eliminated. ${ }^{1-3}$ Success with repeat arthroplasty is probably most strongly related to the virulence and antibiotic resistance characteristics of the organisms.

Chances for success are, for instance, far greater if the organism is quite sensitive to available antibiotics than if this is not true. I have not seen a successful repeat arthroplasty after a documented infection caused hy pseudomonas aeruginosa. The longer the time after prosthetic removal and wound closure, the better the chances for success. ${ }^{2}$ Factors which improve the prospects for a successful repeat arthroplasty include the quality of the debridement with removal of the prosthesis. Retained cement or infected granulation tissue decreases the chances of a successful result. There is considerable enthusiasm for the surgical implantation of an antibiotic-impregnated cement spacer to eliminate dead space and dispense high level concentrations of antibiotics. ${ }^{4}$ Such a spacer 
can be fashioned to preserve the space necessary for the subsequent prosthetic arthroplasty.

Very frequently there will be considerable metaphyseal bone loss by the time of the second implantation and often a customized modular prosthesis will be needed for substitute for, not only lost bone, but also for the stability normally provided by the ligaments and capsule. ${ }^{1}$ With necessary prosthetic constraint comes the need for stems. It must be recognized that if infection complicates the second replacement, stems will cause more bone loss and greater tissue damage.

If the second procedure is done with use of bone cement there is general agreement that a specifically effective heat resistant antibiotic should be added to the bone cement. At the moment, the role of pressfit porous coated components for post-infection revision arthroplasty is not clearly defined.

Rarely does the successful reimplantation prosthetic arthroplasty provide the patient with the satisfaction and functional improvement expected of primary arthroplasty. With the described treatment program authors have reported relatively high success rates, but most studies have a short follow-up period.

\section{Resection Arthroplasty}

Since removal of the prosthesis, joint debridement, antibiotics and external limb support are common to all reasonable programs for treatment of infected total knees, and because any further reconstruction requires a second decision plan and procedure, it has seemed reasonable to sculpt the residual metaphyseal bone ends to fit each other and to use internal and external stabilization to maintain the optimal positions until soft tissue scar function provides a degree of internal linkage.

We have had a limited group of patients who either did not want to risk reimplantation surgery or who had so many medical contraindications that resection arthroplasty remained their final treatment. ${ }^{5}$ For these patients an initial long leg cast was replaced by a long leg brace with knee locks. The brace tended to promote scar flexibility in the direction of normal knee flexion and extension. Up to $40^{\circ}$ of flexion extension motion have been achieved in this way. The brace locks provide complete security in full extension and the false knee motion with the brace unlocked allows for more convenient seated activities. This is particularly advantageous with respect to use of public transportation and social events requiring close seating.

The thigh portion of such a brace can be modified to provide initial weight-bearing such that the limb can have maximum standing security and load-bearing ability while allowing the described seating posture.

Rarely, the resection arthroplasty knee can develop medial-lateral stability and the hamstrings and quadriceps muscles can achieve control of available flexion and extension such that walking and sitting without the brace may be possible. All such patients have, however, used a cane or crutch.
The alternatives of attempt at stemmed prosthesis reimplantation and arthrodesis are always discussed with these patients.

\section{Arthrodesis}

The inconvenience of brace usage: weight, skin problems, clothing wear and the time and effort required for putting the brace on as well as residual pain with motion have provided reasons for a large group of patients to select bone arthrodesis. For a patient with a healed wound that is clinically not infected, we have recommended intramedullary arthrodesis. ${ }^{6.7}$ The procedure requires fresh contact between maximum possible areas of femoral and tibial bone. The tibial shaft is reamed. Minimal femoral reaming is done. A long curved intramedullary rod is inserted at the hip, across the contact region between the femur and tibia, and past the isthmus of the tibia. For 26 such patients we have universally achieved immediate stability without the need for external support. The rod has been sufficiently strong to allow ambulation before bony union.

Functional ankylosis or arthrodesis has been accomplished in all but two patients without the need for bone grafting. There has been no implant failure. The disadvantages of this approach include the magnitude of the surgery, the chances of mechanical failure or fracture of the tibia, and the chances of reinfection. In our experience with 27 cases, the tibial shaft has fractured two times. These fractures have all healed and the complication has not compromised the end result. Two of our patients have developed recurrence of deep infection requiring rod removal. One has required amputation for continued pain and protracted infection at the knee.

\section{Septic Failure}

The absence of infection provides far greater opportunity for the surgeon to salvage failed total knee arthroplasty. The mechanism of failure provides the most useful guide to correction. The wide range of available prosthetic components, the availability of fresh frozen transplant bone, and our increasing experience provide the resources for increasingly successful approaches to these failed knees.

\section{Loosening}

The most frequent cause of aseptic failure of prosthetic knee arthroplasty has been loosening of the components at the prosthesis-bone or cement-bone interface. The tibial component has been most frequently affected. While provision of metal support for the tibial polyethylene, less constrained femoral-tibial interactions and modern cement techniques have decreased the incidence of tibial loosening, this remains the most problematic area.

Revision arthroplasty remains the most frequently 
successful approach to tibial component loosening. The best approach to this problem involves following several important principles. First the procedure must conserve as much as possible of the patient's living bone. The remaining structural soft tissue should be utilized so far as possible for stability. A proper mechanical alignment must be accomplished. Intramedullary stems should be used only when necessary.

The least demanding problem involves a loosened non-stemmed tibial component associated with a secure femoral component and no major soft tissue functional loss. It is obvious that one must predict this situation and have available revision tibial components that can solve the interface failure problem and at the sane time mate geometrically with the retained femoral component. For tibial component revision only the tibial exposure must be circumferential. All bone edges must be seen. And the patellar tendon must not be allowed to tear from the tibial tubercle An extension of the incision into the quadriceps muscle and a preliminary lateral release helps greatly to prevent avulsion at the tubercle.

If there is considerable tibial metaphyseal trabecular bone present revision may be direct without resorting to a stem that extends to the diaphysis. Modern metal wedges are helpful. ${ }^{\circ}$ Occasionally autologous bone grafting may be used. A large mass of cement in an empty metaphysis is rarely successful. If little or no trabecular bone is available, a stemmed prosthesis must be used. I have not encountered the situation of a loose femoral component associated with secure tibial fixation, but would utilize the same principles.

Our experience with revision of one or more aseptic loose components without significant metaphyseal bone loss has been small but generally the results have been very gratifying.

\section{Instability}

A less frequent but more serious failure results from unacceptable joint instability as a result of soft tissue incompetence. This has usually resulted from excessive medial slide procedures and losses of soft tissue competence with excessive exposure. Other cases have involved surgical saw transection of medial collateral ligaments at the primary arthroplasty. We have encountered patients who have had acute trauma to the knee with resultant instability.

While sometimes stability can be achieved by tensing what residual soft tissue remains using thicker tibial components, this grossly affects the joint kinematics and may even result in avulsion of the patellar tendon.

Our greatest experience in treating this situation has been with a long stem intrinsically stable patellofemoral spherocentric prosthesis. ${ }^{9,10}$

Others have used stemmed prostheses with central tibial protrusions that fit into femoral cavities. ${ }^{1.2}$ These different available models of revision knees convey varying degrees of stability depending upon the length and closeness of fit of the protrusion. A few basic principles must be applied here as well. The greater the intrinsic stability of the prosthesis the greater the variety and magnitudes of stress that will be applied to the prosthesis-bone interfaces.

Increasing constraint greatly increases the stresses on the implant itself and thus mechanical failure of the prosthesis becomes more likely. Unsupported structural usage of polyethylene greatly increases the chances of mechanical failure. Thus if a central tibial post is used for medial-lateral and anterior-posterior stability it should be sufficiently strong. A central metal support may be beneficial. The wider the prosthesis the lesser the varus and valgus loads on the prosthetic linkage components. As a general rule the widest prosthesis possible should be used for the trcatment of instability.

\section{Loosening and Instability}

It would seem that instability resulting from grossly loose prosthetic components would be effectively treated by a repeat operation to secure the components in place. In such cases stemmed but relatively unconstrained components should be recommended. In my experience however, the very extensive loss of metaphyseal bone has often destroyed the origins of the knee's major ligaments and simple stemmed revision is insufficient treatment for the resulting instability. If at trial reduction there is still gross instability, a prosthesis which intrinsically augments stability should be used.

\section{Special Problems}

There are additional failure types that are encountered infrequently but are very problematic and difficult for our patients. Fractures above the femoral component occur far more frequently than below the tibial component. Such fractures have, for the most part, been treated by cast immobilization or by open reduction and internal fixation. Usually a condylar blade plate or telescoping screw and side plate are used. While successes have been achieved with bone techniques, they have a common problem in that weight-bearing must be restricted for many weeks. This elderly. systemically ill population responds poorly to enforced inactivity. Under circumstances where a bed to chair life is unacceptable for 12 or more weeks, we have utilized a long-stemmed prosthesis to bypass the fracture and allow for immediate weightbearing walking.

Another very difficult problem is that of avulsion of the patellar tendon from the tibial tubercle. Usually this was at the prior surgery and one is faced with securely fixed components, a high patella and a severe extension lag. I have had limited success with direct suture of the tendon to bone with 8 weeks of cast immobilization in extension. Most such patients require brace control of their limbs. 
The recent introduction of ingrowth stabilized prostheses will present new problems for salvage. Where ingrowth is successful, such components will be difficult to remove. Gentle loosening of the prosthesis/bone interface with a thin saw or osteotome generally allows for component removal with minimal bone loss. I have not encountered the need to remove a component supported by a porous coated stem but experience with such hip prostheses indicates that removal might be impossible without longitudinal sectioning of the bone.

Treatment of aseptic failure of bone arthroplasties really relies on the same foundation as primary arthroplasty. So far as possible the patient's bone should be preserved. The more the stability of the joint can be based on the patient's soft tissue the better. Best functional results involve restoration of a normal joint line location. Normal mechanical alignment, the center of the femoral head, the center of the knee and the center of the ankle lying on a straight line, is essential. The least constrained or securely linked prostheses that will satisfy the mechanical needs the better. All previously used cement and all reactive membrane must be removed whether cement or mechanical fixation is planned for the salvage revision. If cement is to be used, antibiotics in the cement may offer some protection against infection.

Finally, a warning about unexplained pain. Revision surgery should not be performed as an exploratory procedure. Sufficient low risk diagnostic techniques exist including radiography, technetium and gallium or indium radionucleotide scans, aspiration, arthroscopy, and synovial biopsy coupled with investigations of neural and vascular function provide a diagnosis of important pathologies without resorting to exploratory surgery.
While salvage sepsis failure is less satisfactory than salvage for most forms of aseptic knee arthroplasty failure, we must accept the responsibility to provide the best life possible for our patients with both routine and difficult problems.

(Readers should refresh their memories by rereading the Mini-Symposium on Failed Joint Replacement in Current Orthopaedics, Volume 1 Number 1, 1986.)

\section{References}

1. Insall $\mathbf{J} \mathbf{N}$, Thompson $\mathbf{F} \mathbf{N}$, Brause B D. Two-stage reimplantation for the salvage of infected total knee arthroplasty. J Bone Joint Surg 1983; 65 A : $1087-1098$

2. Morrey B F, Westholm F, Schoifet S, Rand J A, Bryan R S. Long-term results of various treatment options for infected total knee arthroplasty. Clin Orthop $1989 ; 248: 120-128$

3. Rand $\mathbf{J} \mathbf{A}$, Bryan R S. Reimplantation for the salvage of an infected total knee arthroplasty. J Bone Joint Surg 1983;65A: 1081-1086.

4. Booth R E, Lotke P A. The results of spacer block technique in revision of infected total knee arthroplasty. Clin Orthop 1989 ; 248: 57-60

5. Falahee M H, Matthews L S, Kaufer H. Resection arthroplasty as a salvage procedure for a knee with infection after total arthroplasty. J Bone Joint Surg 1987; 69A : 10131021 .

6. Harris C M, Froehlich J. Knee Fusion with intramedullary rods for the salvage of infected total knee arthroplasty. Clin Orthop 1985; 197; 209-216.

7. Wilde A H, Stearns K L. Intramedullary Fixation for arthrodesis of the knee after infected total knee arthroplasty. Clinical Orthop 1989; 248: 87-92

8. Brand M G, Daley R J, Ewald F C, Scott R D. Tibial tray augmentation with modular metal wedges for tibial bone stock deficiency. Clin Orthop 1989; 248: 71-79

9. Kaufer H, Matthews L S, Goldstein S A. The long stem spherocentric knee prosthesis for revision of failed total knee arthroplasty. Orthop Trans 1987; 11(1): 168-169

10. Matthews L S, Goldstein S A, Kaufer H. Experiences with three distinct types of total knee joint replacement arthroplasty. Clin Orthop 1985; 192: 97-107 